Variable distraction 1

Running head: VARIABLE DISTRACTION

Altering the flow of mental time: A test of retrieved-context theory

Sean M. Polyn, James E. Kragel, Joshua D. McCluey

Vanderbilt University

John F. Burke

University of Pennsylvania

Sean M. Polyn

Vanderbilt University, Department of Psychology

PMB 407817

2301 Vanderbilt Place

Nashville, TN 37240

e-mail: sean.polyn@vanderbilt.edu

phone: (615) 322-2536

fax: (615) 343-8449

Version of March 10, 2015 
Variable distraction 2

\begin{abstract}
The flexibility and power of the human memory system are exemplified by its ability to create associations between temporally discontiguous events. Retrieved-context theory proposes that these associations are mediated by indirect associations binding the neural representation of each study event to a temporally sensitive contextual representation, which is used as a retrieval cue during memory search. When past states of this contextual representation are reactivated, this gives rise to temporal organization in recall sequences. Previous empirical and simulation work has established that temporal organization is insensitive to the temporal scale of the study experience: Adding substantial periods of inter-item distraction to a study list does not affect the temporal organization of memories for that list. However, this previous work has only examined experiments in which all periods of inter-item distraction in a study list are increased or decreased by an equivalent amount. Here, we demonstrate and test a previously unexamined prediction of retrieved-context theory: The scale-insensitivity of temporal organization is conditional on how temporal structure is manipulated. Specifically, we show that temporal organization is dependent on the relative duration of the temporal intervals surrounding a study event, in a way predicted by retrieved-context theory. Using Bayesian estimation techniques, we demonstrate that the ability of a model to predict the order of responses in free recall is substantially improved when the duration of inter-item distraction intervals influences the representational structure of temporal context. We contrast the predictions of our retrieved-context model with those of an influential model of temporal distinctiveness.
\end{abstract}


Variable distraction 3

\section{Altering the flow of mental time: A test of retrieved-context theory}

The importance of understanding how temporal information is represented in the brain has been recognized since the earliest days of psychological research (Ebbinghaus, 1885/1913; James, 1890). In order to explain time-sensitive psychological phenomena in the domain of learning and memory, theorists have developed computational models in which stimulus representations are associated with a temporally sensitive internal representation (Estes, 1955; Bower, 1972; Mensink \& Raaijmakers, 1988; Levy, 1996; Wallenstein, Eichenbaum, \& Hasselmo, 1998; Howard \& Kahana, 2002; Howard, Shankar, Aue, \& Criss, in press). Estes laid the foundation for these theories in his work on stimulus sampling theory, showing that a model in which associations are formed between items and a slowly changing internal representation can account for major phenomena in

the conditioning literature, including extinction and spontaneous recovery (Estes, 1955). Bower (1972) suggested that a major component of this slowly changing representation could be thought of as reflecting one's inner mental context, the set of thoughts, goals, plans, and intentions that are active when a stimulus is encountered. Bower's conception of inner mental context as an ever-changing entity shares much in common with James' description of the ever-flowing stream of consciousness, the unending cascade of thoughts that make up our experience (James, 1890). Bower used stimulus sampling theory to bring these ideas into contact with experimental data: Under this framework, context is an internal representation that changes gradually as time passes; it is associated with studied material, and can be used as a retrieval cue, to target particular memories that took place in that context. This allowed him to explain a number of time-sensitive behavioral phenomena in both list discrimination paradigms and temporal judgment paradigms.

If inner mental context represents things like passing thoughts, goals, and intentions, 
then this suggests that one can intentionally shift their context, causing events from before the shift to be less memorable. Sahakyan and Kelley (2002) demonstrated this by asking participants to perform an imaginative task during the delay between two study lists (participants imagined what they might do if they had the power of invisibility). This mental activity caused memories for the first list to become less accessible, and memories from the second list to become more accessible, presumably due to reduced proactive interference from the memories of the first list. This suggests that distracting mental activity will cause recently learned things to become less memorable. This has been demonstrated many times in the domain of free recall: A short delay after a list filled with effortful mental activity greatly reduces the recency advantage for end-of-list study items (Postman \& Phillips, 1965; Glanzer \& Cunitz, 1966; Howard \& Kahana, 1999). Petrusic and Jamieson (1978) demonstrated that with increasingly difficult end-of-list distraction tasks, one could progressively dampen memorability of the studied items, to the point where recall of items from all serial positions in a short list were affected.

Retrieved-context models provide a computational framework for understanding how contextual change affects performance on memory tests (Howard \& Kahana, 2002; Sederberg, Howard, \& Kahana, 2008; Polyn, Norman, \& Kahana, 2009a; Lohnas, Polyn, \& Kahana, in press). Many of the ideas proposed by Bower are preserved in these models: As study items are encountered, they are associated with a gradually changing contextual representation. This contextual representation can be used as a retrieval cue during memory search, supporting the retrieval of memories that were associated with similar contextual states. The critical cognitive operation at the heart of these models involves the retrieval of prior states of the contextual representation, a mechanism that wasn't described in Bower's earlier work. In these models, when a particular studied item is recalled, the system also reactivates the contextual state that was associated with that item. This allows the model to perform what Endel Tulving referred to as mental time 
Variable distraction 5

travel, in which one travels backward on their mental timeline, revisiting, in a sense, their past experience (Tulving, 1993).

According to retrieved-context theory, the structure of one's mental timeline greatly influences the structure of one's memories. Organizational analyses of memory propose that the associative structure of memory is reflected in the order in which memories are retrieved in a memory search task like free recall (Puff, 1979; Friendly, 1979; Kahana, 1996). When one remembers a particular item, and reactivates the contextual state associated with the study event, this gives rise to temporal organization: The retrieved contextual information becomes part of one's contextual retrieval cue, causing the memories of the neighboring study events to become more accessible. These dynamics give rise to the contiguity effect of free recall, the general tendency for nearby study events to be recalled successively during memory search. A lag-based conditional response probability analysis reveals the contiguity effect (Kahana, 1996). With this analysis, one calculates the conditional probability of transitions between the studied items, with each transition labeled according to the positional distance (i.e., lag) between the two recalled items. The contiguity effect exhibits itself in terms of the distribution of these lags, with short-lag transitions being much more likely than long-lag transitions, on the whole.

In a retrieved-context model, the contextual representation gradually changes throughout the study period. As each item is studied, it is associated with the current state of the contextual representation. During recall, the context representation acts as a retrieval cue, reactivating the representations of studied items. Polyn et al. (2009a) used a spotlight metaphor to describe the dynamics of contextual cuing. The items are spread across a darkened stage, with the placement of each item indicating its position on the mental timeline defined by the contextual representation. The current state of context determines where the spotlight is pointed; if the list just ended, the spotlight illuminates the last few items on the list, giving rise to the recency effect. When a particular item is 
Variable distraction 6

reactivated, and the context of the study event is retrieved, the spotlight is centered on the just-remembered item. This causes the neighboring items to be illuminated, giving rise to the contiguity effect.

The contiguity effect is quite robust; while several seconds of math distraction at the end of a list is sufficient to disrupt the recency effect, adding the same amount of distraction between each and every study item does not affect contiguity at all (Howard \& Kahana, 1999; Kahana, 2012; Lohnas \& Kahana, 2014). This phenomenon, referred to as long-range contiguity, is challenging for theories of memory in which temporal intervals are bridged by maintaining item information across the delay (Atkinson \& Shiffrin, 1968; Raaijmakers \& Shiffrin, 1981). Under these theories, math distraction at the end of the list should disrupt maintained item information, leading to a diminished recency effect, as is observed. However, when the same math task is interpolated between the study items, this should disrupt the representation of the item preceding the delay. This should lead to diminished associations between neighboring items, and consequently a diminished contiguity effect, which is not observed (Howard \& Kahana, 1999). Sederberg et al. (2008) demonstrated that retrieved-context models can account for the long-range contiguity effect. Under their model, the distracting mental activity disrupts the contextual representation by causing it to change. Going back to the spotlight metaphor, this is like evenly increasing the spacing between all of the items on the stage, as depicted schematically in Figure 1a. When the spotlight is centered on a just-recalled item, the illumination of both of its neighbors decreases by a similar amount. Recall is a competitive process, so the relative likelihood of recalling either of these items next is unchanged. Even when the length of the inter-item distraction intervals are doubled from 8 to 16 seconds, the shape of the contiguity effect is unchanged (Lohnas \& Kahana, 2014).

Retrieved-context theory proposes that while the contiguity effect is insensitive to this manipulation, the memory system itself is quite sensitive to the amount of inter-item 
distraction. Each distraction interval perturbs the representation of temporal context, causing the mental timeline to advance. Evenly increasing these distraction intervals does not alter temporal organization, because the relative support for surrounding items is unchanged. However, retrieved-context theory makes a heretofore untested prediction: One should be able to experimentally manipulate the shape of the contiguity effect, by changing the durations of these inter-item distraction intervals in an uneven manner. Figure $1 \mathrm{~b}$ depicts this in terms of the spotlight analogy.

Here, we provide empirical support for this previously untested prediction of retrieved-context theory. Using a retrieved-context modeling framework, we demonstrate that the relative durations of the distraction intervals on a study list influence the organization of one's memories, suggesting that the content of these intervals are important in determining the structure of one's mental timeline. These results also bear upon an older debate in the memory literature, regarding the influence of a distraction task on the memorability of studied material. Briefly, Koppenaal and Glanzer (1990) and Thapar and Greene (1993) demonstrated that if a participant must shift from one distraction task to another during a study period, this task shift can decrease the memorability of items studied before the shift. These results raise the possibility that the negative consequences of distraction arise simply because a person must shift from an encoding task to a distraction task; in this scenario the relative duration of distraction intervals may be unimportant in determining item memorability and recall organization. Our modeling framework allows us to address this question directly, and we return to this issue in the discussion.

Finally, these results bear upon temporal distinctiveness theories of human memory, under which the memorability of a particular item is affected by its temporal distance from the recall test, as well as the item's temporal distance from other study events. In order to qualitatively contrast retrieved-context models with temporal distinctiveness 
models, we examine the memorability of items as a function of the duration of the surrounding distraction intervals. In this experiment, temporally isolated items are less well remembered than temporally crowded items, a finding which may be at odds with the predictions of temporal distinctiveness models. We consider this finding in terms of both our retrieved-context model and an influential model of temporal distinctiveness, the Scale-Invariant Memory, Perception, and Learning model (SIMPLE; Brown, Neath, \& Chater, 2007).

Insert Figure 1 about here

\section{Materials and Methods}

\section{Participants}

40 participants (18 female), aged 18-27, were tested in accordance with University of Pennsylvania IRB guidelines and were paid for their time. Participants were tested as part of a longer series of free-recall experiments, and as such, were familiar with both the free-recall paradigm and with the orienting tasks used in the experiment. The majority of the participants (32) performed 4 sessions of the experiment. One participant performed 2 sessions, and 6 performed 5 or more sessions (with a maximum of 8 sessions, for two of the participants). For each of the 6 participants performing more than 4 sessions, we examined their performance on sessions 5 through 8 on all the major summary statistics reported in this manuscript. We found no evidence of any systematic changes in their behavioral performance between the first four sessions and these later sessions. As such, we decided to include all sessions in the analyses reported in this manuscript. 
Variable distraction 9

\section{Materials and list creation}

1655 words were drawn from the University of South Florida free association norms, based on whether each word was appropriate for the two orienting tasks used in the experiment, described below.

Procedure

During each session a participant completed 10 trials of the paradigm. On each trial, participants studied a list of 15 words drawn from the word pool without replacement (words were allowed to repeat across sessions). Each word was presented for 3 seconds (followed by a blank screen that varied in duration from 800-1200 msec); within this time participants were asked to make a 2 choice judgment with a keypress (either a size judgment: "Is this item bigger or smaller than a shoebox?", or an animacy judgment: "Does this word refer to something living or nonliving?"). In any given session all words were judged with the same orienting task.

The items on the list were preceded and followed by interstimulus intervals (ISIs) that ranged from 6 seconds to 23 seconds (including the intervals before the first and after the final item). During these ISIs, participants engaged in a mental arithmetic task. A three-term arithmetic problem of the form $A+B+C=$ ? was presented, and participants made a keyboard response. A counter at the top left of the screen during the arithmetic task kept the participant's score (incrementing by 1 if a correct response was given). Participants were instructed that their performance on the arithmetic task would influence a bonus payment.

On fixed distraction trials, these ISIs were always $11.76 \mathrm{sec}$. On variable distraction trials the ISIs followed the schedule: $(6.00,8.40,11.76,16.46,23.05,23.05,16.46,11.76$, $8.40,6.00 \mathrm{sec})$. Each variable distraction trial started from a random point on this schedule, and looped to the beginning upon reaching the final element (see Fig. 2a for a 
schematic of a variable distraction trial). As such, there were 10 distinct variable distraction schedules, each representing a different starting point on the schedule. After the distraction period following the final item (drawn from the same schedule of ISIs), a set of asterisks and a beep indicated the start of a free-recall period, in which the participant had 90 seconds to verbally recall the studied items in any order.

Insert Figure 2 about here

\section{Simulation methods}

\section{A likelihood-based retrieved-context model}

We used a retrieved-context model of free recall, the Context Maintenance and Retrieval - Likelihood (CMR-L) model, as part of an analytic framework designed to test a prediction of retrieved-context theory regarding the interaction of inter-item distraction and the contiguity effect. Under this framework the model can be used in two ways: First, the model can be used to predict the likelihood of individual recall events, allowing us to characterize model fitness in terms of the likelihood of a given model having given rise to the full set of recall events across all trials in the experiment. Second, the model can be used to generate synthetic recall sequences, allowing us to determine whether the best-fitting model can properly account for the major summary statistics observed in these data.

In prior work, retrieved-context models have been evaluated in terms of their ability to generate recall sequences whose summary statistics match those of the observed data (e.g., Sederberg et al., 2008; Polyn et al., 2009a; Lohnas et al., in press). In the current work, the goodness-of-fit of a given model is calculated in terms of the model's ability to predict the full set of recall events recorded in the experiment (Kragel, Morton, \& Polyn, 
2015; Morton \& Polyn, n.d.). The recall sequence of the participant is coded as a series of recall events, with one event for each response made by the participant, and a final event representing the termination of memory search. In this sense, each recall event can be thought of as a selection from a categorical distribution containing $\mathrm{k}$ items (one for each of the not-yet-recalled study items, and one for recall termination), where the model determines the probability of selecting each of these items. The model's prediction regarding the actual response is appended to a vector of likelihood values $\left(p_{\text {event }}\right)$, where each element of the vector corresponds to particular recall event. The likelihood of the model producing the full set of experimental data is the product of all elements in the $p_{\text {event }}$ vector. Given the vast number of possible recall sequences, this is a very small number; following convention, we represent this likelihood using log-transformed probability values, as follows:

$$
L=-\sum \log \left(p_{\text {event }}\right)
$$

where $L$ represents the overall fitness of the model in terms of these data, with a smaller value indicating a more fit model.

\section{The structure and dynamics of the CMR-L model}

Basic structure of the model. The model is a simplified neural network, with two representational sub-layers $F$ and $C$, corresponding respectively to a feature-based representation of studied material, and an internal contextual representation. Each item is represented by a unit vector $\mathbf{f}(i)$ on $F$, with $i$ indexing list position. The state of context at each list position corresponds to a vector $\mathbf{c}(i)$ on $C$. Two associative matrices $\mathbf{M}^{F C}$ and $\mathbf{M}^{C F}$ determine the interactions between $F$ and $C$. The matrix $\mathbf{M}^{F C}$ is initialized to represent any pre-experimental associations between $F$ and $C$. A parameter $\gamma$ controls the relative strength of these pre-experimental associations and the associations formed over 
the course of the study period. The diagonal elements of $\mathbf{M}^{F C}$ represent the pre-experimental associations from $F$ to $C$, and are initialized at $1-\gamma$.

Contextual integration during the study period. As each item is presented, the item's representation $\mathbf{f}(i)$ is activated. This representation is projected through $\mathbf{M}^{F C}$, giving rise to $\mathbf{c}^{\mathrm{IN}}$, which is the input to $C . C$ contains integrative machinery that causes its state, $\mathbf{c}(i)$, to change gradually over the course of the list, as follows:

$$
\mathbf{c}(t)=\rho \mathbf{c}(t-1)+\beta \mathbf{c}^{\mathrm{IN}}(t),
$$

where

$$
\mathbf{c}^{\mathrm{IN}}(t)=\mathbf{M}^{F C} \mathbf{f}(t),
$$

and

$$
\rho(t)=\sqrt{1+\beta^{2}\left[\left(\mathbf{c}(t-1) \cdot \mathbf{c}^{\mathrm{IN}}(t)\right)^{2}-1\right]}-\beta\left(\mathbf{c}(t-1) \cdot \mathbf{c}^{\mathrm{IN}}(t)\right) .
$$

When an item is presented, the parameter controlling integration rate, $\beta$, is set to a value $\beta_{\text {enc }}$.

Prior to the presentation of the first list item, context is initialized to the state $\mathbf{c}_{\text {start }}$, by activating the unit vector $\mathbf{f}_{\text {start }}$ and allowing this start-of-list representation to completely update the contextual representation, following Equation 2, with $\beta$ set to 1 .

Contextual integration during the math distraction intervals. Before and after each study item, the participant performs a distraction task. Thus, each list has 16 distraction intervals, one preceding the first item, and one following each of the 15 studied items. The distraction intervals change in duration over the course of the list, with the duration of a given distraction interval (in milliseconds) given by $x(d)$, where $d$ indexes 
the distraction interval. Each distraction interval is represented as a unit vector $\mathbf{f}(d)$. Following the simulation methods of Sederberg et al. (2008), the distraction task disrupts the contextual representation by causing it to integrate this non-item-related information.

The amount of disruption is determined by the contextual integration rate $\beta_{\text {dist }}$, which is a linear function of the distraction interval duration $x(d)$, as follows:

$$
\beta_{\text {dist }}=m x(d)+b
$$

where $b$ is a parameter controlling the baseline contextual integration rate in the presence of distraction, and $m$ is a slope parameter controlling how sensitive the contextual integration rate is to the duration of the distraction interval. The contextual representation is then updated as described in Equation 2, with $\beta$ set to the value $\beta_{\text {dist }}$.

The $\beta_{\text {dist }}$ parameter is constrained to the interval [ $\left[\begin{array}{ll}0 & 1\end{array}\right]$. In order to ensure that the values chosen for $m$ and $b$ during the parameter optimization process did not cause $\beta_{\text {dist }}$ to stray from this interval, we introduced a parameter $b_{i n i t}$, which was used to determine an appropriate value of $b$. The parameter $b_{\text {init }}$ was chosen from the interval [0 1]. For a given value of $m, b_{\text {init }}$ was linearly mapped onto an interval that ensured that $\beta_{\text {dist }}$ itself stayed within the interval [ $\left[\begin{array}{ll}0 & 1\end{array}\right]$. If $b_{\text {init }}$ was set to 0 , then a value of $b$ was chosen such that the shortest distraction interval (6000 milliseconds) corresponded to a $\beta_{\text {dist }}$ of 0 . If $b_{\text {init }}$ was 1 , then $b$ was chosen such that the longest distraction interval (23050 milliseconds) corresponded to a $\beta_{\text {dist }}$ of 1 .

Association formation during the study period. After each item is presented, and the contextual representation is updated, the item representation and the contextual representation become associated to one another by a simple Hebbian learning rule, as follows: 


$$
\begin{aligned}
& \mathbf{M}^{F C}(t+1)=\mathbf{M}^{F C}(t)+\gamma \mathbf{c}(t) \mathbf{f}(t)^{\top} \\
& \mathbf{M}^{C F}(t+1)=\mathbf{M}^{C F}(t)+\phi(t) \mathbf{f}(t) \mathbf{c}(t)^{\top},
\end{aligned}
$$

where $\gamma$ represents the learning rate of experimental associations and ${ }^{\top}$ denotes the outer product operation. In order to account for the enhanced memorability of items from the beginning of the study list (i.e., the primacy effect), we included an associative gradient mechanism represented by $\phi(t)$, following the simulation methods of Sederberg et al. (2008) and Polyn et al. (2009a):

$$
\phi(t)=\phi_{s} e^{-\phi_{d}(t-1)}+1
$$

where $\phi(t)$ takes on a value $\phi_{s}+1$ for the first list position, and decays exponentially towards 1 with a rate determined by $\phi_{d}$. This associative learning mechanism is only engaged when an item is presented, it is not engaged during the distraction intervals. We note that in our first round of parameter estimation, the best-fitting value of the $\phi_{s}$ parameter was effectively 0 , suggesting that this primacy mechanism is ineffective in producing a primacy effect that matches the observed data.

Simulating the recall period. After the final distraction interval, the recall period begins. As mentioned above, the recall period is simulated as a sequence of recall events, where a recall event can either indicate successful recall of a particular item, or termination of the search. The last event of any recall sequence is always the termination event. The end-of-list contextual state is used as a retrieval cue, to reactivate the features of studied material on $F$, following:

$$
\mathbf{s}(t)=\mathbf{M}^{C F} \mathbf{c}(t)
$$


During the recall period $t$ indexes recall events, rather than list position. The vector s is activated on F; as described by Howard and Kahana (2002), it is a blend of item features, similar to many but identical to none. This blended representation fuels a recall competition, with two possible outcomes: Reactivation of the representation of a particular studied item, or recall failure, and termination of memory search. The probability of recall termination for any given recall event is given by $p_{\text {stop }}$, which is calculated as follows:

$$
p_{\text {stop }}=\xi_{s}+e^{-\xi_{d} S_{n r} / S_{r}} .
$$

Here, $\xi_{s}$ represents the probability of terminating search without recalling any items, and $\xi_{d}$ controls how $p_{\text {stop }}$ increases with output position, scaling the ratio $S_{n r} / S_{r}$, where $S_{n r}$ is the sum of the elements of s corresponding to the not-yet-recalled items, and $S_{r}$ is the sum of the elements of $\mathbf{s}$ corresponding to the already recalled items. Since each item is represented as a unit vector on $F$, the non-zero element on $F$ for that item can be considered as corresponding to that item.

Once the probability of recall termination is calculated, a probabilistic choice rule is used to simulate a competitive recall process between the remaining not-yet-recalled studied items. The probability of recalling each of the remaining studied items, $p_{t}$ (here, $t$ indexes list position), is calculated according to:

$$
p_{t}=\left(1-p_{\text {stop }}\right) \frac{e^{2 \mathbf{s}_{t} / \tau}}{\sum_{i=1}^{N} e^{2 \mathbf{s}_{i} / \tau}},
$$

where $\tau$ controls the ferocity of the competition; as $\tau$ gets smaller, the probability scores approach those of a winner-take-all rule.

Under the generative version of the model, these probabilities are used to randomly determine whether the recall event will correspond to recall of a particular item, or recall termination. If an item is recalled, the item's representation is reactivated on $F$. This 
representation is used to update the contextual representation, following Equation 2, with $\beta$ set to the value $\beta_{\text {rec }}$. Then the next recall event begins, as described above, until a termination event is chosen or all items are recalled.

Under the likelihood version of the model, the probability corresponding to the actual response of the participant is selected and appended to the vector $p_{\text {event }}$. If an item is recalled, the item's representation is reactivated on $F$, context is updated, and recall continues, as described above.

A context-based primacy mechanism. Our initial simulations with these data revealed that the associative gradient primacy mechanism used by Sederberg et al. (2008) and Polyn et al. (2009a) was inadequate to account for the primacy effect in these data. This motivated us to include a second primacy mechanism, inspired by other computational models of free recall (Laming, 1999; Davelaar, Goshen-Gottstein, Ashkenazi, Haarmann, \& Usher, 2005). Under this mechanism, the memory system automatically reactivates a small amount of the start-of-list contextual state prior to each recall attempt. Before each recall competition, the start-of-list representation $\mathbf{f}_{\text {start }}$ is reactivated, and the contextual representation is updated following Equation 2, with $\beta$ set to the value $\beta_{\text {start }}$. In our preliminary work with the model, we found that regardless of whether one or both primacy mechanisms were included as part of the model, the conclusions regarding the relationship between inter-item distraction and temporal organization were unchanged.

\section{Parameter estimation and model predictions}

Parameter names, allowable ranges, best-fitting values, and highest posterior density intervals are provided in Table 1. A particle swarm optimization (PSO) technique was used to find the best-fitting model (Eberhart \& Kennedy, 1995). An adaptive Metropolis-Hastings Markov Chain Monte Carlo (MCMC) technique was used to estimate 
the posterior distribution of model parameters, given the observed data. We initialized the MCMC algorithm with the best-fitting set of parameters found by the PSO. A burn-in period of 3000 iterations was used to ensure the convergence of the chains, followed by sampling of 5000 parameter sets. Uninformative prior distributions were constructed for each parameter of interest, consisting of a Gaussian distribution with mean equal to the maximum likelihood parameter estimates and infinite standard deviation. Parameter values were bounded at the ranges described in Table 1.

In order to characterize the influence of inter-item distraction on recall organization, we used the CMR-L framework to generate a large number of synthetic recall sequences. For each synthetic trial, we randomly sampled a parameter set from the posterior distribution identified by the MCMC. In order to gain a sense of the variability in the data, we labeled these model-generated recall sequences as coming from 40 simulated participants. For each simulated participant, we generated 25 recall sequences for each possible distraction schedule, yielding a total of 1000 trials with each possible distraction schedule. These model-generated recall sequences were used to compare the model and the observed data in terms of a set of summary statistics describing recall initiation, recall transitions, overall memorability of studied items, and recall termination.

Preliminary work with the CMR-L model suggested that the associative gradient primacy mechanism used by Sederberg et al. (2008) and Polyn et al. (2009a) was less effective than a context-based primacy mechanism (both mechanisms are described above). These preliminary results motivated us to remove the associative gradient mechanism from the model. The estimated posterior distributions for the various model parameters were similar regardless of whether the associative gradient mechanism was included, including for the critical parameter $m$. 


\section{Simulation of item memorability using SIMPLE}

We simulated the temporal distinctiveness model SIMPLE using code provided by G. Brown, including the correction described by Lee and Pooley (2013). The PSO described above was used to find the best-fit parameter set. Because SIMPLE does not predict recall organization, we could not use the same likelihood-based fitting technique as used for CMR-L. Following previous work with SIMPLE, we fit the model to a number of summary statistics, minimizing a $\chi^{2}$ statistic comparing observed statistics to model-generated statistics. These statistics included probability of recall by serial position (15 data-points), as well probability of recall as a function of distraction condition: peak, trough, rising, and falling (4 data-points).

SIMPLE has 4 parameters: $c$ controls the rate at which similarity falls of with distance. $\alpha$ controls the relationship between temporal distance and similarity in the model. If this parameter is set to 1 , this relationship is exponential, if it is set to 2 , this relationship is Gaussian. We performed two searches, one for each possible $\alpha$ setting. The final two parameters, $s$ and $t$, are specific to simulations of free recall, and control the shape of a transforming function that allows for items from a particular list position to be correctly recalled even when the model is probing a different list position (in serial recall, this would result in an error). The reader is referred to Brown et al. (2007) and Lee and Pooley (2013) for a fuller description of the dynamics of SIMPLE.

\section{Results}

\section{Likelihood-based optimization of CMR-L}

The best-fitting model from the PSO algorithm was used as the starting point for an MCMC algorithm, designed to estimate the posterior distributions of the model parameters, given these data. Geweke's convergence diagnostic indicated that the MCMC chain had converged on a stationary posterior distribution (all $p>0.7$ ). Table 1 reports 
the possible range and best-fitting value for each free parameter of the model, as well as 95\% highest density intervals for the posterior distributions estimated by MCMC. The mean fitness $L$ for all models accepted as part of the MCMC chain was 33308; $L$ for the best-fitting model was 33300 ; the $95 \%$ highest density interval on the distribution of $L$ was [33302-33317].

Contextual disruption due to distraction is ultimately controlled by the parameter $\beta_{\text {dist }}$, which is not itself a free parameter, but is controlled by $m$ and $b_{\text {init }}$, as described. Of primary interest was the posterior distribution of model parameter $m$, which represents the slope of the linear mapping between distraction interval duration and contextual disruption. Because interval duration was coded in milliseconds, the allowable values of $m$ were numerically small. Given the best-fitting values of $m$ and $b$ reported in Table 1 , the $\beta_{\text {dist }}$ parameter ranged from 0.48 (for the shortest distraction intervals) to 0.65 (for the longest). An examination of the posterior distribution of $m$ revealed that all of the models in the MCMC chain had $m>0$. In other words, the family of models providing a good fit to these data all required the contextual integration process to be influenced by the length of the inter-item distraction interval. Recall organization is reliably influenced by inter-item distraction, in these data.

Insert Table 1 about here

In the following sections we examine the performance of the model, and compare this with the observed behavioral performance across a set of summary statistics describing the influence of the temporal structure of the study list on recall organization and memorability of the studied material. 
Variable distraction 20

Temporal organization and recall transitions

Insert Figure 3 about here

In order to characterize the relationship between the temporal structure of the study list and the temporal organization of the studied materials in memory, we examined the sequential properties of participants' recall responses. Figure 3a shows a lag-CRP curve for all list positions (i.e., regardless of the amount of distraction surrounding a given item). We observed a strong contiguity effect in these data: Participants tended to successively recall items that were studied in neighboring positions on the list. This replicates findings by Howard and Kahana (1999) and Lohnas and Kahana (2014) that even though studied items are separated in time by sizable periods of distracting mental

activity (arithmetic), neighboring items still tend to be recalled successively. The lag-CRP curve also exhibited a forward asymmetry: Forward-going recall transitions were more likely than backward-going transitions (Kahana, 1996; Howard \& Kahana, 1999; Kahana, Howard, \& Polyn, 2008).

Retrieved-context theory predicts that the length of the distraction intervals surrounding a particular studied item will have a reliable influence on the temporal organization of that item in the recall sequence. Specifically, the theory predicts that the forward asymmetry of the contiguity effect will be attenuated under conditions of rising distraction. In order to test this prediction, we created a statistic sensitive to the magnitude of the forward asymmetry. This asymmetry score specifies the relative likelihood of forward-going and backward-going recall transitions. To calculate this score, we took the average probability of a forward-going transition to a nearby item (lags +1 and +2 ) and subtracted from this the average probability of a backward-going transition to a nearby item (lags -1 and -2 ). We partitioned the recall transitions into two sets. In 
the first set, we included any recall transition initiated from an item studied under conditions of falling distraction. Transitions in the second set were initiated from items studied under conditions of rising distraction. An asymmetry score was calculated for each distraction condition.

As can be seen in Figure 3b, the prediction that forward-going transitions will be attenuated under conditions of rising distraction is borne out in the current experiment. The shift in the asymmetry effect is significant by a one-tailed $t$-test $(t(38)=2.9, p<0.005)$. Figure $3 \mathrm{~b}$ also presents the model predictions regarding the magnitude of this effect; even though the model was fit to individual recall events, it does an excellent job capturing the shift in asymmetry ( $t$-test on simulated data: $t(39)=3.39, p<0.005)$. This effect is apparent even if the set of valid recall transitions is restricted to only include transitions between items from mid-list serial positions 6-10, thus avoiding any potential influence of primacy or recency effects (mean asymmetry score and $[\mathrm{SEM}])$ : falling $=0.063[0.019]$, rising $=0.025[0.018]$, significant by a one-tailed $t$-test: $t(38)=1.92, p<0.05$.

Temporal isolation and the memorability of studied material

Insert Figure 4 about here

Figure 4a shows the probability of recall by serial position for this experiment, across all list types, for both observed data and simulated recall sequences. The shallow (relative to immediate free recall) primacy and recency effects are representative of results obtained with previous continual distraction free-recall (CDFR) paradigms (Bjork \& Whitten, 1974; Tzeng, 1973; Koppenaal \& Glanzer, 1990; Howard \& Kahana, 1999; Polyn, Norman, \& Kahana, 2009b). The model does a reasonable job capturing the shape of the serial 
position curve, though it slightly underpredicts both the primacy and recency effects.

In order to characterize the effect of the temporal structure of the list on item memorability, we constructed the two serial position curves presented in Figure 4b, one for items studied under conditions of peak distraction (23 sec on either side) and one for items studied under conditions of trough distraction (6 sec on either side). Generally speaking, items studied under peak distraction are remembered more poorly than those studied under trough distraction, regardless of serial position, an effect that is captured by the model (Fig. 4c).

Insert Figure 5 about here

In order to better characterize the relationship between memorability and distraction, we partitioned study items according to where they fell on the distraction schedule, and calculated the proportion of items recalled from each set (as shown in Figure 5). To avoid the influence of primacy and recency effects, we restricted this analysis to items from the flat portion of the serial position curve (positions 6-10). Items studied at the peak of the distraction schedule are significantly worse recalled than items at the trough of the distraction schedule $(t(38)=-2.19, p<0.05)$, and the model captures this effect $(t(39)=-5.2, p<0.005)$. In other words, items that are temporally crowded on the timeline of the study list are better recalled than those that are temporally isolated.

\section{A simulation of item memorability using SIMPLE}

Our finding that temporally crowded items are better recalled than temporally isolated items can be contrasted with results reported by Brown, Morin, and Lewandowsky (2006), in which temporally isolated items were better recalled than crowded items during free recall. The temporal distinctiveness model SIMPLE (Brown et 
al., 2007) has been used to understand the influence of the temporal structure of study lists on the memorability of studied items. In SIMPLE, each item, as it is studied, is given a position on a mental timeline. Memory retrieval is cast as a problem of discriminability; memories that are near one another on the mental timeline can interfere with one another. These neighboring memories are confusable; when one probes for a particular memory, other nearby memories may be retrieved instead.

SIMPLE can be used to predict recall performance in both free recall and serial recall. In serial recall, SIMPLE simulates the recall period as a series of recall attempts, where each serial position is probed in turn. The extension of SIMPLE to free recall uses this same probing mechanism; in consequence, SIMPLE does not predict the organization of responses in free recall, although it can be used to generate predictions regarding the likelihood of a given item being recalled. As such, in this section, we examine the ability of SIMPLE to account for item memorability in this experiment.

In the case of serial recall, SIMPLE makes a clear prediction: Temporally isolated items should be recalled more accurately than temporally crowded items. If the model probes for an item in one serial position but retrieves an item studied in another serial position, this is an error in serial recall, as the items must be reported in the order that they were studied. In the case of free recall, the predictions regarding the relationship between item memorability and temporal structure are less clear. In free recall, as long as an item is recalled at some point in the recall sequence, it is counted as correct, regardless of the order of responses. In other words, in free recall, the confusability of temporally proximate items can end up being beneficial for recall performance, as a given item has multiple chances to be recalled. Without explicitly fitting SIMPLE to our data, it is unclear whether our observation of an advantage for temporally crowded items is challenging for this model.

We fit SIMPLE model to the data from the current experiment, as described in the 
methods; as mentioned, we fit two versions of SIMPLE to the data, one in which similarity decreases exponentially with increasing distance $(\alpha=1)$ and another in which this decrease is described by a Gaussian $(\alpha=2)$. For the exponential model, best-fitting parameters were $c=4.27, s=0.776, t=4.34\left(\chi^{2}(16)=19.54\right)$. For the Gaussian model, best-fitting parameters were $c=9.44, s=0.826, t=4.03\left(\chi^{2}(16)=21.34\right)$. Each model produced a reasonable qualitative fit to the serial position curve, though like CMR-L each underpredicted the primacy effect. However, neither version of SIMPLE was able to qualitatively capture the relationship between item memorability and the amount of surrounding distraction for mid-list serial positions. Best-fit predictions for peak and trough items for the exponential model: 0.518 and 0.517 respectively. For the Gaussian model: 0.516 and 0.517 . These values can be contrasted with the corresponding values in the observed data, peak: $0.467(S E M=0.04)$, trough: $0.517(S E M=0.04)$, as presented in Figure 5. In summary, despite the potential for SIMPLE to capture an advantage for temporally crowded items in free recall, it was unable to qualitatively fit the results of the current experiment.

\section{General Discussion}

The indirect item-to-context associations of retrieved context theory provide an account of the basic cognitive mechanisms allowing the mammalian memory system to span short time-scales (Rawlins, 1985; Bunsey \& Eichenbaum, 1996; Hilgard \& Bower, 1966; Howard et al., in press). Under retrieved context theory, these same mechanisms allow for flexible search of the long-term associative structures of the brain (Polyn \& Kahana, 2008; Howard, Fotedar, Datey, \& Hasselmo, 2005; Polyn et al., 2009a). The theory has important characteristics in common with computational models of rodent memory, which describe temporally sensitive populations of hippocampal cells that bridge temporal delays in conditioning studies and support sequence learning (Levy, 1996; 
Wallenstein et al., 1998). Suggestive evidence for such temporally sensitive cells has been found in rodent hippocampus (MacDonald, Lepage, Eden, \& Eichenbaum, 2011), a structure critically important for association formation. Characterization of this neural machinery could potentially unify species-spanning time-sensitive phenomena such as extinction and spontaneous recovery (Bouton, 2002), classical conditioning across temporally discontiguous events (Rawlins, 1985; Shankar \& Howard, 2012), and transitive inference (Bunsey \& Eichenbaum, 1996; Howard, Jing, Rao, Provyn, \& Datey, 2009). Retrieved context theory makes explicit predictions regarding what the neural correlates of temporal context should look like, both in terms of the autocorrelative structure of the signal and its relation to behavior (Polyn \& Kahana, 2008). Recent work showing the functional importance of slow changes in neural activity patterns in both rodents and humans (Manns, Howard, \& Eichenbaum, 2007; Jenkins \& Ranganath, 2010; Manning, Polyn, Baltuch, Litt, \& Kahana, 2011) points to a new generation of computational models that define the cognitive mechanisms underlying these neural signals, and how they give rise to the behavioral phenomena of memory (Kragel et al., 2015).

A handful of prior studies have demonstrated that the shape and magnitude of the contiguity effect is insensitive to manipulations of inter-item distraction when the relative spacing of the items is unchanged (Howard \& Kahana, 1999; Lohnas \& Kahana, 2014). Here, we present empirical work showing that when the relative spacing of items is altered by allowing distraction interval duration to vary within list, there is a reliable change in the shape of the contiguity effect, in a way predicted by context-based theories, but not other theories of memory. CMR-L provides a mechanistically explicit instantiation of retrieved-context theory, allowing us to precisely quantify the consequences of this distraction on the organization of memories. The critical mechanism determining how inter-item distraction influences temporal context was proposed by Sederberg et al. (2008), but the prediction regarding the effect of within-list changes in distraction on 
recall organization was previously untested.

In retrieved-context theory, the memory system spans discontiguous temporal intervals by binding items to an ever-changing representation of temporal context. This can be contrasted with alternative buffer models in which an item representation is itself maintained across a temporal interval, and then directly associated with the next item (Atkinson \& Shiffrin, 1968; Raaijmakers \& Shiffrin, 1981). Theoretical work by Howard and Kahana (1999) suggests that buffer models are challenged by the long-range contiguity effect: In short, if end-of-list distraction disrupts the buffer, and thereby recency (as is observed), the same amount of inter-item distraction should disrupt the buffer, and thereby contiguity (which is not observed).

It is worth noting that it is possible for a dual-store model to accomodate the long-range contiguity effect by incorporating a context-based retrieval mechanism with dynamics similar to those described by retrieved-context models. Davelaar et al. (2005) present such a hybrid model. The contextual representation slowly changes its state (drifts) during inter-item distraction periods, but since this drift is gradual, neighboring list items still tend to be associated with similar states of context. Because the contextual representation is used to probe memory during the recall period, the association of items to similar states of context gives rise to the long-range contiguity effect.

\section{Contextual disruption and the distraction task}

The parsimony of a context-based model over a buffer-based model (without a temporal context representation) relies on the idea that distraction effectively prevents rehearsal, making direct inter-item associations impossible. Koppenaal and Glanzer (1990) challenged this idea with a proposal that participants can adapt to a distraction task given some practice on it, and then engage in a form of time-sharing between distraction-task performance and maintenance of studied items in a buffer. They carried 
out a continual distraction free recall experiment in which the identity of the distraction task changed in the retention interval following the presentation of the final study item. With this manipulation, the long-term recency effect was abolished, consistent with the idea that while participants had adapted to the first distraction task, the new distraction task was unfamiliar, disrupting the time-sharing process, and emptying the buffer of recently studied items.

Thapar and Greene (1993) challenged this interpretation by showing that the amount of practice or familiarity that a participant has with a distraction task does not influence the degree that task disrupts the recency effect in either delayed or continual distraction free recall paradigms (see also, Neath, 1993). These experiments ruled out the simple distraction adaptation hypothesis put forth by Koppenaal and Glanzer (1990), but raised a question that has not been adequately addressed, regarding the nature of the disruptive effects of distraction tasks on a participant's ability to maintain or rehearse a particular studied item (and thereby create direct associations between items). Specifically, these experiments raised the possibility that the distraction task per se is not the source of disruption, but rather, the act of shifting from one distraction task to another that is disruptive (of either a buffer or a contextual representation).

The current work directly contrasts these two hypotheses. When the CMR-L model parameter $m$ is greater than 0 , this represents the hypothesis that the performance of the distraction task itself is disruptive to context: the magnitude of the disruption scales with the amount of time spent performing the distraction task. If $m$ was equal to 0 , this represents the alternative hypothesis that each distraction interval is equally disruptive (as controlled by the $b$ intercept parameter), as would be the case if the act of shifting to the distraction task was disruptive, and not the amount of time spent performing the task. Our Bayesian parameter estimates were unequivocal in their support of models with $m>0$. In other words, knowing the length of a distraction interval was important for the 
model's ability to predict the organization of recall.

\section{Isolation, memorability, and temporal distinctiveness}

The family of temporal-distinctiveness models were explicitly designed to account for the effects of stimulus timing and temporal intervals on the memorability of studied material, and were in part motivated by the difficulty faced by buffer models in explaining the long-term recency effect (Glenberg et al., 1980; Glenberg, Bradley, Kraus, \& Renzaglia, 1983). The SIMPLE model (Brown et al., 2007) formalizes temporal distinctiveness theory in a computational model that has been applied to both serial recall and free recall phenomena. While SIMPLE can generate predictions regarding the memorability of items based on the temporal structure of the list, it does not explicitly model the order of responses during memory search. Thus, it makes no predictions regarding the effect of inter-item distraction on the long-range contiguity effect.

We found that the results of this experiment challenge a temporal distinctiveness model: SIMPLE was unable to capture the increased memorability of temporally crowded items (trough distraction) relative to temporally isolated items (peak distraction) observed in this experiment. At the heart of SIMPLE is a mechanism that causes items that are temporal neighbors to interfere with one another. It seems in this scenario, the best SIMPLE can do to fit the current data is to predict no difference in memorability based on the relative length of surrounding distraction intervals. We have not carried out a quantitative comparison of CMR-L and SIMPLE, because the many differences between the structure of the two models (and the way each was fit to the data) make it unclear what model comparison statistic would be appropriate. However, we can still ask the question: Putting aside issues of relative model complexity, what is it about CMR-L that allows it to do a qualitatively better job fitting the results from this experiment?

The critical distinction between the two models is most apparent when one 
considers the recall process in each model. In both models, items become less accessible in proportion to how far they have receded into the past. For SIMPLE, this gradual recession of memories along a mental timeline is inexorable. For CMR-L, it is not, due to the retrieved-context operation at the heart of the model. When CMR-L retrieves context from a past experience, it jumps back along its mental timeline, making the neighbors of the just-remembered item more accessible. It is for this reason that Howard and Kahana (1999) referred to the contiguity effect as the lag recency effect: Retrieved context causes temporal proximity to become advantageous, since the neighbors of the just-remembered item are better supported if they are associated with highly similar states of the temporal context representation. These dynamics allow CMR-L to simultaneously account for the effects of the temporal structure of a study experience on the organization and memorability of studied material. 


\section{References}

Atkinson, R. C., \& Shiffrin, R. M. (1968). Human memory: A proposed system and its control processes. In K. W. Spence \& J. T. Spence (Eds.), The psychology of learning and motivation (Vol. 2, pp. 89-105). New York: Academic Press.

Bjork, R. A., \& Whitten, W. B. (1974). Recency-sensitive retrieval processes in long-term free recall. Cognitive Psychology, 6, 173-189.

Bouton, M. E. (2002). Context, ambiguity, and unlearning: Sources of relapse after behavioral extinction. Biological Psychiatry, 52(10), 976-986.

Bower, G. H. (1972). Stimulus-sampling theory of encoding variability. In A. W. Melton \& E. Martin (Eds.), Coding processes in human memory (pp. 85-121). New York: John Wiley and Sons.

Brown, G. D. A., Morin, C., \& Lewandowsky, S. (2006). Evidence for time-based models of free recall. Psychonomic bulletin \& review, 13(4), 717.

Brown, G. D. A., Neath, I., \& Chater, N. (2007). A temporal ratio model of memory. Psychological Review, 114(3), 539-576.

Bunsey, M., \& Eichenbaum, H. B. (1996). Conservation of hippocampal memory function in rats and humans. $379(6562), 255-257$.

Davelaar, E. J., Goshen-Gottstein, Y., Ashkenazi, A., Haarmann, H. J., \& Usher, M. (2005). The demise of short-term memory revisited: Empirical and computational investigations of recency effects. Psychological Review, 112, 3-42.

Ebbinghaus, H. (1885/1913). On memory: A contribution to experimental psychology. New York: Teachers College, Columbia University.

Eberhart, R., \& Kennedy, J. (1995, October). A new optimizer using particle swarm theory. In Proceedings of the sixth international symposium on micro machine and human science, 1995. MHS '95 (pp. 39-43).

Estes, W. K. (1955). Statistical theory of spontaneous recovery and regression. 
Psychological Review, 62, 145-154.

Friendly, M. (1979). Methods for finding graphic representations of associative memory structures. In C. R. P. (Ed.) (Ed.), Memory organization and structure (pp. 85-129). New York: Academic Press.

Glanzer, M., \& Cunitz, A. R. (1966). Two storage mechanisms in free recall. Journal of Verbal Learning and Verbal Behavior, 5, 351-360.

Glenberg, A. M., Bradley, M. M., Kraus, T. A., \& Renzaglia, G. J. (1983). Studies of the long-term recency effect: Support for a contextually guided retrieval theory. Journal of Experimental Psychology: Learning, Memory \& Cognition, 12, 413-418.

Glenberg, A. M., Bradley, M. M., Stevenson, J. A., Kraus, T. A., Tkachuk, M. J., \& Gretz, A. L. (1980). A two-process account of long-term serial position effects. Journal of Experimental Psychology: Human Learning and Memory, 6, 355-369.

Hilgard, E. R., \& Bower, G. H. (1966). Theories of learning (3rd ed.). New York: Appleton-Century-Crofts.

Howard, M. W., Fotedar, M. S., Datey, A. V., \& Hasselmo, M. E. (2005). The temporal context model in spatial navigation and relational learning: Toward a common explanation of medial temporal lobe function across domains. 112(1), 75-116.

Howard, M. W., Jing, B., Rao, V. A., Provyn, J. P., \& Datey, A. V. (2009). Bridging the gap: Transitive associations between items presented in similar temporal contexts. Journal of Experimental Psychology: Learning, Memory, and Cognition, 35(2), $391-407$.

Howard, M. W., \& Kahana, M. J. (1999). Contextual variability and serial position effects in free recall. Journal of Experimental Psychology: Learning, Memory, 8 Cognition, 25, 923-941.

Howard, M. W., \& Kahana, M. J. (2002). A distributed representation of temporal context. Journal of Mathematical Psychology, 46, 269-299. 
Howard, M. W., Shankar, K. H., Aue, W. R., \& Criss, A. H. (in press). A distributed representation of internal time. Psychological Review.

James, W. (1890). The principles of psychology. New York, NY, US: Henry Holt and Co, Inc.

Jenkins, L. J., \& Ranganath, C. (2010). Prefrontal and medial temporal lobe activity at encoding predicts temporal context memory. Journal of Neuroscience, 30(46), $15558-15565$.

Kahana, M. J. (1996). Associative retrieval processes in free recall. Memory $\&$ Cognition, 24, 103-109.

Kahana, M. J. (2012). Foundations of human memory (1st ed.). New York, NY: Oxford University Press.

Kahana, M. J., Howard, M. W., \& Polyn, S. M. (2008). Associative retrieval processes in episodic memory. In H. L. Roediger III (Ed.), Cognitive psychology of memory. Vol. 2 of Learning and memory: A comprehensive reference, 4 vols. (J. Byrne, Ed.) (pp. 467-490). Oxford: Elsevier.

Koppenaal, L., \& Glanzer, M. (1990). An examination of the continuous distractor task and the long-term recency effect. Memory \& Cognition, 18, 183-195.

Kragel, J. E., Morton, N. W., \& Polyn, S. M. (2015, February). Neural Activity in the Medial Temporal Lobe Reveals the Fidelity of Mental Time Travel. The Journal of Neuroscience, 35(7), 2914-2926.

Laming, D. (1999). Testing the idea of distinct storage mechanisms in memory. International Journal of Psychology, 34, 419-426.

Lee, M. D., \& Pooley, J. P. (2013). Correcting the simple model of free recall. Psychological Review, 120(1), 293-296.

Levy, W. B. (1996). A sequence predicting CA3 is a flexible associator that learns and uses context to solve hippocampal-like tasks. Hippocampus, 6, 579-590. 
Lohnas, L. J., \& Kahana, M. J. (2014). Compound cuing in free recall. Journal of Experimental Psychology: Learning, Memory, and Cognition, 40(1), 12-24.

Lohnas, L. J., Polyn, S. M., \& Kahana, M. J. (in press). Expanding the scope of memory search: Modeling intralist and interlist effects in free recall. Psychological Review.

MacDonald, C. J., Lepage, K. Q., Eden, U. T., \& Eichenbaum, H. (2011). Hippocampal "time cells" bridge the gap in memory for discontiguous events. Neuron, 71(4), $737-749$.

Manning, J. R., Polyn, S. M., Baltuch, G., Litt, B., \& Kahana, M. J. (2011). Oscillatory patterns in temporal lobe reveal context reinstatement during memory search. Proceedings of the National Academy of Sciences of the United States of America, $108(31), 12893-12897$.

Manns, J. R., Howard, M. W., \& Eichenbaum, H. (2007). Gradual changes in hippocampal activity support remembering the order of events. Neuron, 56(3), 530-540.

Mensink, G.-J. M., \& Raaijmakers, J. G. W. (1988). A model for interference and forgetting. Psychological Review, 95, 434-455.

Morton, N. W., \& Polyn, S. M. (n.d.). A predictive framework for evaluating models of semantic organization in free recall. submitted.

Neath, I. (1993). Contextual and distinctive processes and the serial position function. Journal of Memory and Language, 32, 820-840.

Petrusic, W. M., \& Jamieson, D. G. (1978). Differential interpolation effects in free recall. Journal of Experimental Psychology: Human Learning and Memory, 4(1), 101-109.

Polyn, S. M., \& Kahana, M. J. (2008). Memory search and the neural representation of context. Trends in Cognitive Sciences, 12, 24-30.

Polyn, S. M., Norman, K. A., \& Kahana, M. J. (2009a). A context maintenance and retrieval model of organizational processes in free recall. Psychological Review, $116(1), 129-156$. 
Polyn, S. M., Norman, K. A., \& Kahana, M. J. (2009b). Task context and organization in free recall. Neuropsychologia, 47, 2158-2163.

Postman, L., \& Phillips, L. W. (1965). Short-term temporal changes in free recall. Quarterly Journal of Experimental Psychology, 17, 132-138.

Puff, C. R. (1979). Memory organization research and theory: The state of the art. In C. R. Puff (Ed.), Memory organization and structure (pp. 3-17). New York: Academic Press.

Raaijmakers, J. G. W., \& Shiffrin, R. M. (1981). Search of associative memory. Psychological Review, 88, 93-134.

Rawlins, J. N. P. (1985). Associations across time: The hippocampus as a temporary memory store. Behavioral and Brain Sciences, 8(3), 479-528.

Sahakyan, L., \& Kelley, C. M. (2002). A contextual change account of the directed forgetting effect. 28(6), 1064-1072.

Sederberg, P. B., Howard, M. W., \& Kahana, M. J. (2008). A context-based theory of recency and contiguity in free recall. Psychological Review, 115(4), 893-912.

Shankar, K. H., \& Howard, M. W. (2012). A scale-invariant internal representation of time. Neural Computation, 24, 134-193.

Thapar, A., \& Greene, R. L. (1993). Evidence against a short-term- store account of long-term recency effects. Memory \& Cognition, 21, 329-337.

Tulving, E. (1993). What is episodic memory? Current Directions in Psychological Science, 2(3), 67-70.

Tzeng, O. J. L. (1973). Positive recency in delayed free recall. Journal of Verbal Learning and Verbal Behavior, 12, 436-439.

Wallenstein, G. V., Eichenbaum, H., \& Hasselmo, M. (1998). The hippocampus as an associator of discontiguous events. Trends in Neurosciences, 21(8), 317-323. 
Variable distraction 35

\begin{abstract}
Author Note
This research was supported by National Institutes of Health Grant MH55687 to Michael Kahana and National Science Foundation grant 1157432 to SMP. We gratefully acknowledge the support of Michael Kahana for making this project possible; Richard Lawrence, Gennady Erlikhman, Jonathan Miller, Neal Morton, and Emily Rosenberg, for assistance with data collection and initial analyses of the data; Saul Sternberg, Richard Shiffrin, and Randolph Blake for valuable comments and discussion.
\end{abstract}


Variable distraction 36

\begin{tabular}{lccc}
\hline & Range during optimization & Best-Fit & $95 \%$ HPD interval \\
\hline$\beta_{\text {enc }}$ & $0-1$ & 0.77 & $0.74-0.80$ \\
$\beta_{\text {rec }}$ & $0-1$ & 0.96 & $0.94-0.97$ \\
$\beta_{\text {start }}$ & $0-1$ & 0.25 & $0.21-0.29$ \\
$\gamma$ & $0-1$ & 0.78 & $0.73-0.83$ \\
$\xi_{d}$ & $0-20$ & 6.11 & $5.02-6.95$ \\
$\tau$ & $0.1-1$ & 0.52 & $0.50-0.55$ \\
$m$ & $0-0.00004$ & 0.0000101 & $0.0000062-0.000017$ \\
$b_{\text {init }}$ & $0-1$ & 0.58 & $0.44-0.67$ \\
\hline
\end{tabular}

Table 1

Results of MCMC estimation of posterior distributions on model parameters. HPD: highest posterior density. Fixed parameters include: $\xi_{s}=0.001, \phi_{s}=0, \phi_{d}=0$ 


\section{Figure Captions}

Figure 1. The spotlight analogy of the retrieved-context theory of memory search. When the item "window" is recalled, the context corresponding to that moment is retrieved, which causes the spotlight to shine on the representation of that item, somewhat illuminating neighboring list items. (a) Consider two study lists, one with shorter inter-item distraction intervals (dashed line) and one with longer inter-item distraction intervals (dotted line). In this case the amount of inter-item distraction is increased evenly throughout the list, so given that "window" is recalled, the relative likelihood of recalling the neighboring items is unchanged. (b) Consider now a study list where the relative duration of inter-item distraction intervals changes within-list. In this case, the competitive nature of recall will cause the item that is better illuminated by the spotlight to be favored in the recall competition. If the just-recalled item is on the rising portion of the distraction schedule, items preceding the just-recalled item will be better illuminated and more likely to be recalled next, counteracting the forward asymmetry of the contiguity effect.

Figure 2. Schematic of a representative trial of the variable distraction paradigm. A participant studies a series of 15 items (white ellipses), example items are shown below the timeline. Between items, the participant engages in a distracting task (mental arithmetic; black shading), example problems are shown below the timeline. During a 90-sec. post-list period the participant verbally recalled the studied items in any order.

Figure 3. (a) The contiguity effect in free recall, calculated across all list positions. Each point represents the conditional probability that, after the participant recalls item $N$, the next recalled item will be $N+1(\operatorname{Lag} 1), N-1(\operatorname{Lag}-1)$, etc., conditional on the availability of that item for recall. Successive recall events tend to come from nearby list positions. (b) Altering the temporal structure of the study experience alters the contiguity 
effect. When an item is studied under conditions of rising distraction, forward-going recall transitions become less likely, and backward-going recall transitions become more likely, reducing the forward asymmetry of the contiguity effect. Error bars indicate standard error of the mean, across participants.

Figure 4. (a) Probability of recall by serial position, across all list types. (b) In the behavioral data, items studied under conditions of peak distraction are less memorable than those studied under conditions of trough distraction. (c) The model captures the basic relationship between memorability and the length of the surrounding distraction intervals.

Figure 5. Probability of recall for mid-list serial positions, with studied items partitioned according to where they occurred on the distraction schedule. Circles correspond to behavioral data (with errorbars indicating SEM), and squares correspond to model performance. 

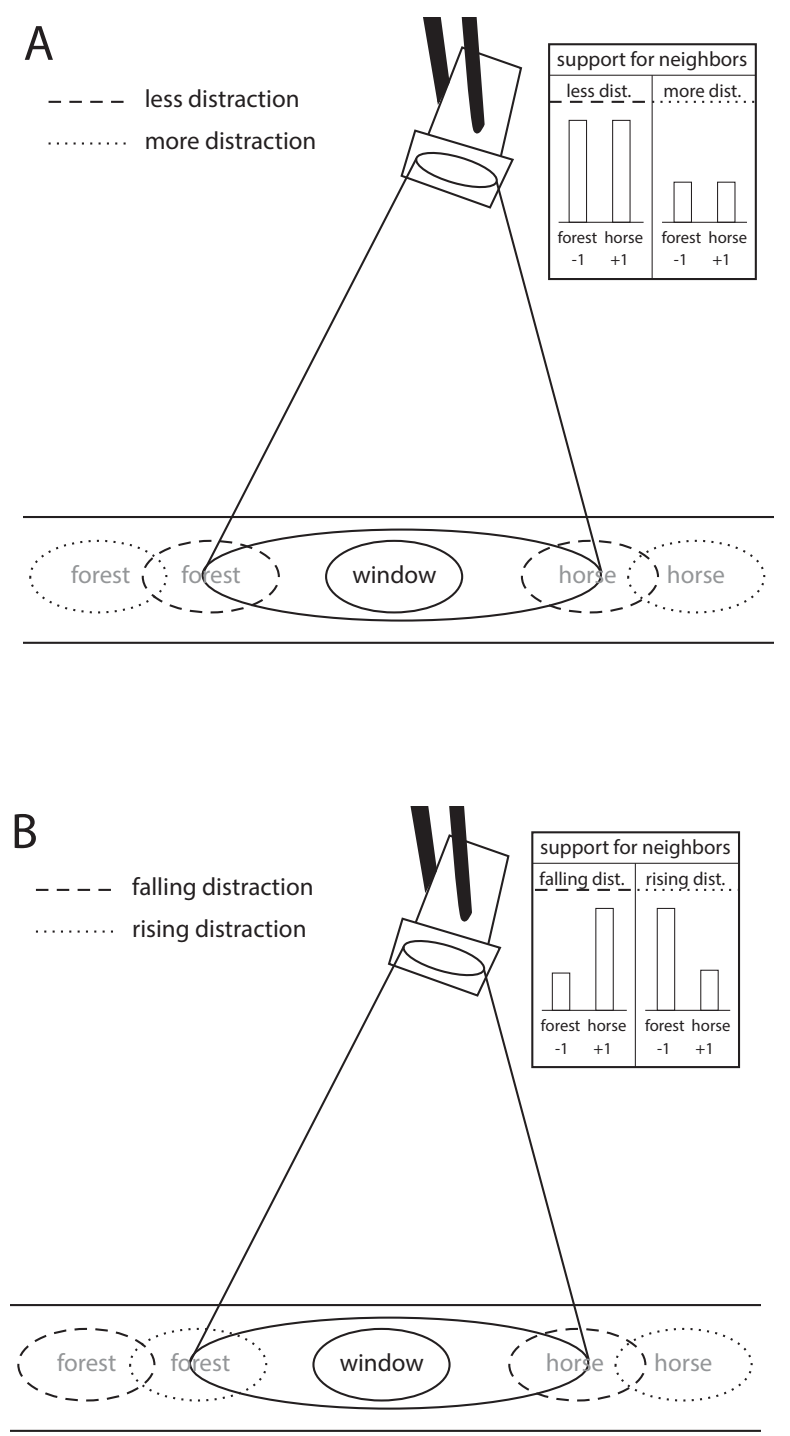


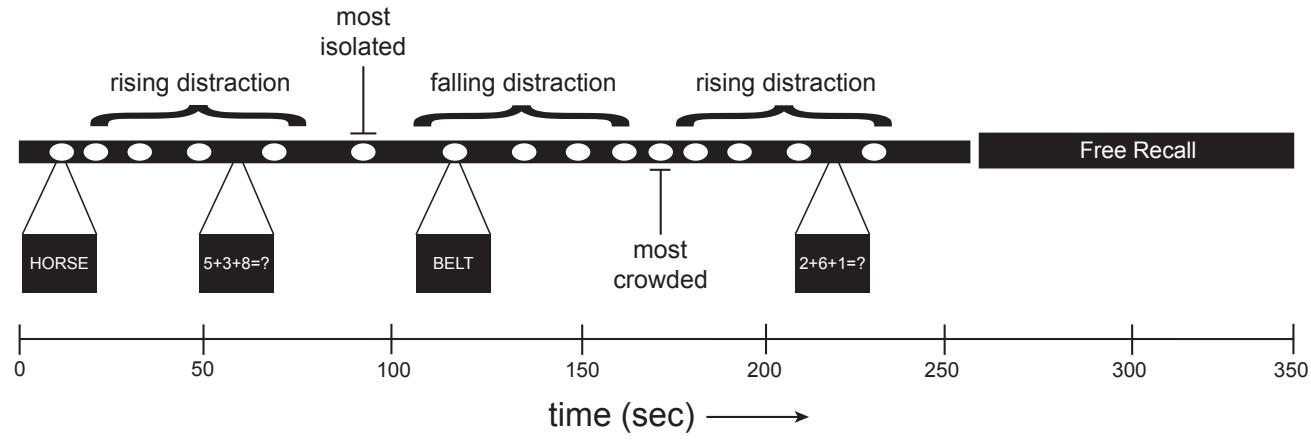


A

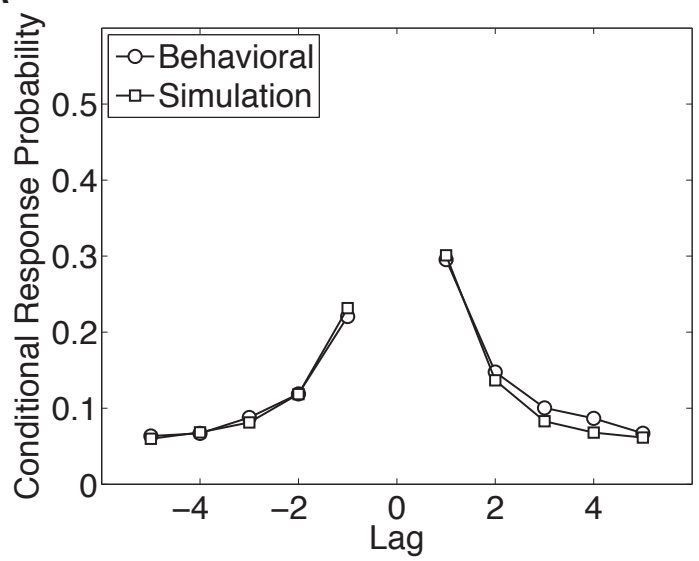

B

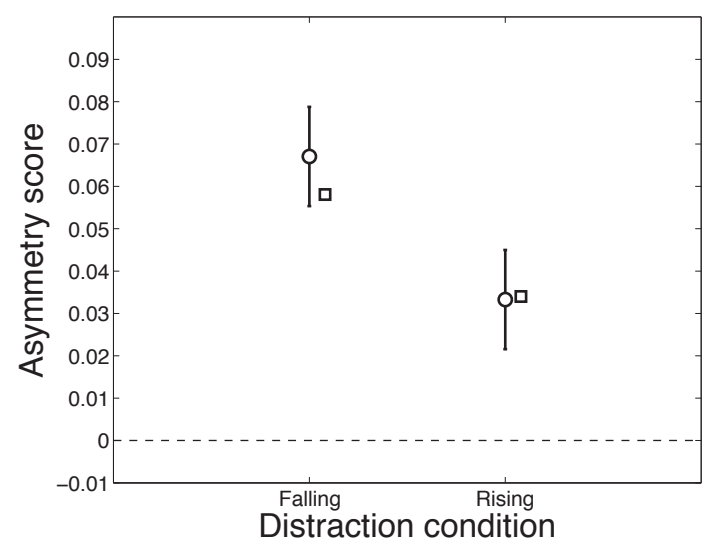


A

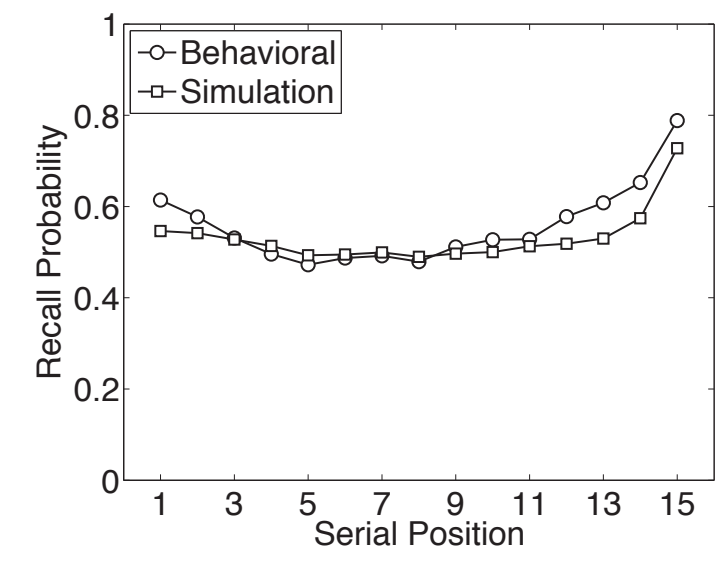

B

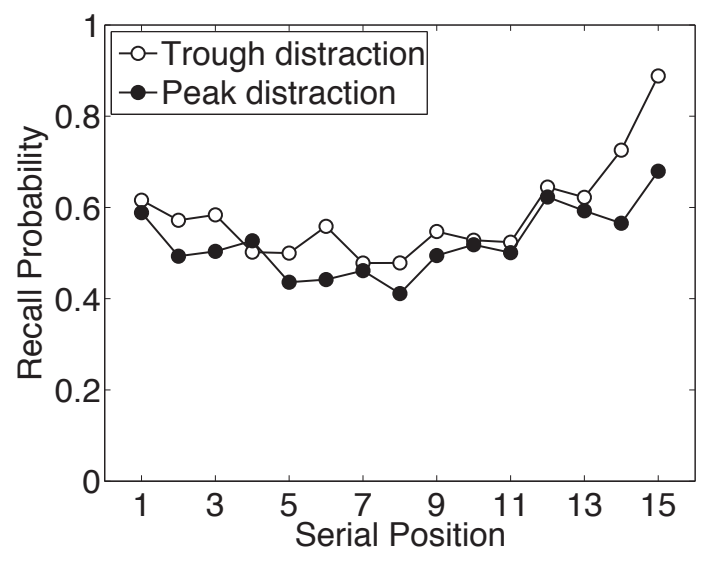

C

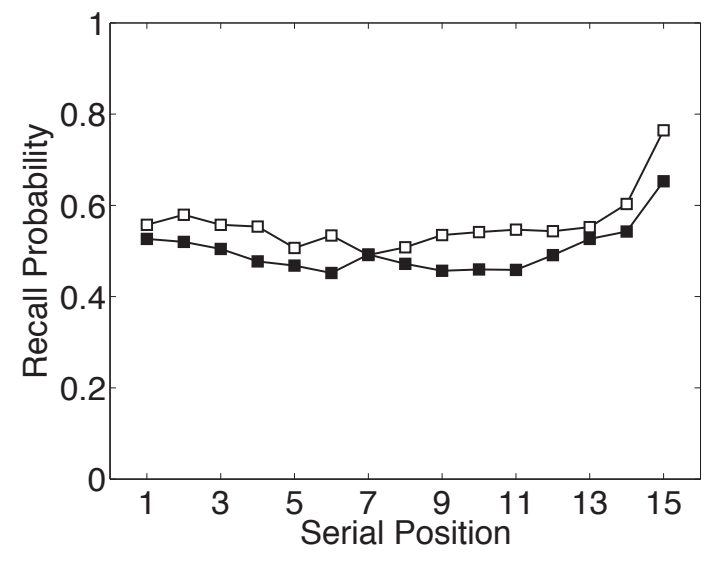




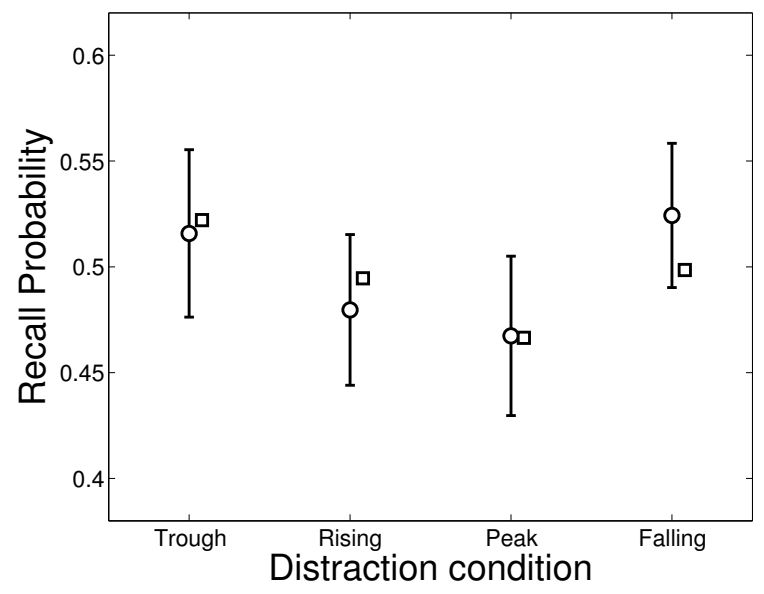

